\title{
Keynote: PhD Recipes
}

\author{
Milad Shokouhi \\ Applied Researcher \\ Microsoft \\ Cambridge, UK \\ milads@microsoft.com
}

\begin{abstract}
Doing research is a bit like cooking. Most people follow similar steps but the outcome is often very different. Each of us create our own special recipes along the way, and eventually we write our very own PhD cookbook. In this talk, I'll share my recipes. Bon Appétit!
\end{abstract}

Keywords: PhD Research, Experiences, Tips 